\title{
Impact of Molecular Subtype Conversion of Breast Cancers after Neoadjuvant Chemotherapy on Clinical Outcome
}

\author{
Siew Kuan Lim, MD ${ }^{1,2}$ \\ Moo Hyun Lee, MD ${ }^{1}$ \\ In Hae Park, MD ${ }^{1}$ \\ Ji Young You, MD ${ }^{1}$ \\ Byung-Ho Nam, $\mathrm{PhD}^{3}$ \\ Byeong Nam Kim, RN 1 \\ Jungsil Ro, MD, $\mathrm{PhD}{ }^{1}$ \\ Keun Seok Lee, MD, PhD ${ }^{1}$ \\ So-Youn Jung, $M D^{1}$ \\ Young Mee Kwon, MD, PhD' \\ Eun Sook Lee, MD, PhD ${ }^{1}$
}

${ }^{1}$ Center for Breast Cancer, Research Institute and Hospital,

National Cancer Center, Goyang, Korea,

${ }^{2}$ Breast Service,

Department of General Surgery,

Changi General Hospital, Singapore,

${ }^{3}$ Cancer Biostatistics Branch,

Research Institute for

National Cancer Control and Evaluation,

Research Institute and Hospital,

National Cancer Center, Goyang, Korea

\section{Purpose}

The aim of this study was to examine molecular subtype conversions in patients who underwent neoadjuvant chemotherapy (NAC) and analyze their clinical implications.

\section{Materials and Methods}

We included consecutive breast cancer patients who received NAC at the National Cancer Center, Korea, between August 2002 and June 2011, and had available data on estrogen receptor (ER), progesterone receptor (PR), and human epidermal growth factor 2 (HER2) receptor status prior to NAC. Molecular subtypes, hormone receptor (HR) status, and ER and PR Allred scores before and after NAC were compared, and the long-term outcomes were analyzed.

\section{Results}

Of 322 patients, 32 (9.9\%) achieved a pathologic complete response after NAC. HR+/HER2 - tumors tended to convert into triple negative (TN) tumors (10.3\%), whereas $34.6 \%$ of TN tumors gained HR positivity to become HR+/HER2- tumors. Clinical outcomes of molecular subtype conversion groups were compared against patients who remained as HR+/HER2 - throughout. The HR+/HER2 - to TN group had significantly poorer recurrencefree survival (RFS) (hazard ratio, 3.54; 95\% confidence interval [Cl], 1.60 to 7.85) and overall survival (OS) (hazard ratio, 3.73; 95\% Cl, 1.34 to 10.38). Patients who remained TN throughout had the worst outcomes (for RFS: hazard ratio, 3.70; 95\% Cl, 1.86 to 7.36; for OS: hazard ratio, $5.85 ; 95 \% \mathrm{Cl}, 2.53$ to 13.51 ), while those who converted from TN to HR+/HER2showed improved comparable survival outcomes.

\section{Conclusion}

Molecular subtypes of breast cancers changed frequently after NAC, resulting in different tumor prognostication. Tumor subtyping should be repeated after NAC in patients with breast cancer.

Center for Breast Cancer,

National Cancer Center, 323 Ilsan-ro,

Ilsandong-gu, Goyang 10408, Korea

Tel: 82-31-920-1503

Fax: 82-31-920-1511

E-mail: eslee@ncc.re.kr

Key words

Breast neoplasms, Molecular subtype, Receptor status,

Received September 22, 2014

Accepted February 15, 2015

Published Online April 7, 2015

\section{Introduction}

Neoadjuvant chemotherapy (NAC) is widely accepted as the standard of care for locally advanced breast cancer. Studies have confirmed that NAC can be used to downsize oper- able breast tumors, both to increase the rate of breast conservation in patients eligible for mastectomy and to achieve better cosmetic results after breast conserving surgery, without compromising long-term outcomes of breast cancer treatment [1-3]. Accordingly, the rate of NAC use has shown a considerable increase. However, there is concern that hor- 
mone receptor (HR) and human epidermal growth receptor 2 (HER2) receptor status can be altered by NAC [4,5]. While some studies reported no change, others have demonstrated changes in estrogen receptor (ER), progesterone receptor (PR), and HER2 receptor status, as described in a recent review by van de Ven et al. [5]. Switching to a positive HR status after NAC was also reported to indicate a better prognostic outcome [6,7].

ER, PR, and HER2 status are well-established prognostic and predictive biomarkers. Patients with a positive HR status have better long-term outcomes whereas those with a positive HER2 status have a poorer prognosis. Endocrine therapy significantly improves the prognosis of patients with HR expression, and prolonged duration of endocrine therapy is now reported to show benefit [8]. Similarly, anti-HER2 targeted therapy in HER2 positive patients significantly improves the long-term outcomes of patients with a HER2 positive status [9]. Tumor responses to chemotherapy also differ according to the expression of these biomarkers, and these responses, in turn, are predictive of recurrence-free survival (RFS) and overall survival (OS) [10]. Therefore, decisions regarding both neoadjuvant and adjuvant therapy and the regimens employed are heavily dependent on the HR and HER2 status of the tumor.

Genomic expression profiling of breast cancers brought about a new classification for breast cancers, with the most common subtypes being luminal A and B, HER2 and basallike types. This classification enables better prognostication, and may further refine the choice of therapy for individual patients [11]. Although genomic profiling is still not performed routinely for each patient, due to its high cost and the need for technical expertise, immunohistochemical (IHC) analyses of protein biomarkers can be used to determine the approximate molecular category [12-14]. Onitilo et al. [14] reported that molecular subtype classification according to ER, PR, and HER2 status provided useful prognostic and therapeutic information on the four major molecular subtypes (luminal A, luminal B, HER2, and basal-like/ triple negative $[\mathrm{TN}])$.

The aim of this study was to examine the changes in IHCbased molecular subtype in patients who underwent NAC at our institution. We also analyzed the patients according to their HR status and changes in their ER and PR Allred scores, and examined the impact of these changes on longterm outcomes.

\section{Materials and Methods}

\section{Patients}

We retrospectively reviewed our database for records of consecutive patients who underwent NAC at National Cancer Center, Korea, between August 2002 and June 2011.

Diagnostic core biopsies were performed before NAC in all patients. Patients for whom data on HR and HER2 status were available were included in the analysis. Those with metastatic disease at diagnosis, bilateral breast cancers, and those who had received any previous treatment for breast cancer were excluded.

\section{HR and HER2 status determination and molecular sub- type grouping}

Paraffin sections of the tumors were subjected to IHC analyses to determine the ER, PR, and HER2 status. ER expression was assessed using the antibody clones 6F11 or SP1, while PR expression was examined using the antibody clones 1A6 or 1E2 (Ventana Medical Systems, Tucson, AZ). Reactivity of more than $1 \%$ was defined as positive for both receptors. ER and PR expression were also quantified using the Allred scoring system. Patients with ER and/or PR positivity were classified as HR positive.

The HER2 status was determined using rabbit polyclonal antibodies (Dako, Glostrup, Denmark). HER2 overexpression was defined as membrane staining of $3+($ HER2 +$)$, while $1+$ and 0 were defined as HER2 negative (HER2-). Fluorescent in situ hybridization (FISH) was performed when the HER2 IHC scores were equivocal $(2+)$, and also when the physician in charge wanted to confirm the IHC results. FISH was performed using the PathVysion Kit (Abbott/Vysis Laboratories, Abbott Park, IL), and HER2 gene amplification was defined as HER2:chromosome 17 ratio of $\geq 2$.2. Whenever FISH tests results were available, they took precedence over the IHC results. Tumors without FISH testing despite an equivocal IHC score were excluded from this study.

Using the above receptors' status, patients were divided into four molecular subtype groups for analyses: (1) HR+ and HER2-, (2) HR+ and HER2+, (3) HR- and HER2+, and (4) HR- and HER2- or TN.

\section{Treatments}

As many of the patients were also participants in various clinical trials of NAC that were being conducted at the National Cancer Centre, Korea, during this period, the NAC regimens were not uniform for all patients $[15,16]$. The regimens included four cycles of doxorubicin and cyclophos- 
Table 1. Patient and tumor characteristics according to molecular subtype at diagnosis

\begin{tabular}{|c|c|c|c|c|c|}
\hline Characteristic & HR+/HER2- & HR+/HER2+ & HR-/HER2+ & $\mathrm{TN}$ & p-value \\
\hline \multicolumn{6}{|l|}{ Histologic type $\left.(\mathrm{n}=317)^{\mathrm{a}}\right)$} \\
\hline Invasive ductal carcinoma & $157(95.2)$ & $64(100)$ & $35(100)$ & $53(100)$ & \multirow[t]{2}{*}{0.056} \\
\hline Invasive lobular carcinoma & $8(4.8)$ & 0 & 0 & 0 & \\
\hline \multicolumn{6}{|l|}{ Age $\left.(y r)(n=322)^{a}\right)$} \\
\hline$<45$ & $79(47.3)$ & $29(44.6)$ & $9(25.7)$ & $23(41.8)$ & \multirow[t]{2}{*}{0.134} \\
\hline$\geq 45$ & $88(52.7)$ & $36(55.4)$ & $26(74.3)$ & $32(58.2)$ & \\
\hline \multicolumn{6}{|l|}{ Clinical stage $\left.(\mathrm{n}=328)^{\mathrm{a}}\right)$} \\
\hline Stage II & $44(27.3)$ & $17(27.0)$ & $5(14.3)$ & $10(19.2)$ & \multirow[t]{2}{*}{0.300} \\
\hline Stage III & $117(72.7)$ & $46(73.0)$ & $30(85.7)$ & $42(80.8)$ & \\
\hline \multicolumn{6}{|l|}{ Grade of tumor $(\mathrm{n}=293)^{\mathrm{a})}$} \\
\hline Grade 1 or 2 & $93(62.4)$ & $32(51.6)$ & $9(27.3)$ & $14(28.6)$ & \multirow[t]{2}{*}{$<0.001$} \\
\hline Grade 3 & $56(37.6)$ & $30(48.4)$ & $24(72.7)$ & $35(71.4)$ & \\
\hline \multicolumn{6}{|l|}{$\mathrm{Ki}-67(\%)^{\mathrm{b})}(\mathrm{n}=283)^{\mathrm{a})}$} \\
\hline$<15$ & $107(70.4)$ & $35(62.5)$ & $10(40.0)$ & $16(32.0)$ & \multirow[t]{2}{*}{$<0.001$} \\
\hline$\geq 15$ & $45(29.6)$ & $21(37.5)$ & $15(60.0)$ & $34(68.0)$ & \\
\hline \multicolumn{6}{|l|}{ Lymphatic invasion $\left.{ }^{b}(n=279)^{a}\right)$} \\
\hline Yes & $132(86.8)$ & $47(92.2)$ & $21(80.8)$ & $46(92.0)$ & \multirow[t]{2}{*}{0.376} \\
\hline No & $20(13.2)$ & $4(7.8)$ & $5(19.2)$ & $4(8.0)$ & \\
\hline \multicolumn{6}{|l|}{ Vascular invasion $^{\mathrm{b})}(\mathrm{n}=271)^{\mathrm{a})}$} \\
\hline Yes & $4(2.7)$ & $2(4.0)$ & 0 & $3(6.4)$ & \multirow[t]{2}{*}{0.473} \\
\hline No & $144(97.3)$ & $48(96.0)$ & $26(100)$ & $44(93.6)$ & \\
\hline \multicolumn{6}{|l|}{ Response to $\mathrm{NAC}^{\mathrm{c}}(\mathrm{n}=310)^{\mathrm{a})}$} \\
\hline Yes & $102(63.4)$ & $44(71.0)$ & $31(88.6)$ & $30(57.7)$ & \multirow[t]{2}{*}{0.013} \\
\hline No & $59(36.6)$ & $18(29.0)$ & $4(11.4)$ & $22(42.3)$ & \\
\hline
\end{tabular}

Values are presented as number (\%). HR, hormone receptor; HER2, human epidermal growth factor 2; TN, triple negative; NAC, neoadjuvant chemotherapy. ${ }^{a} \mathrm{n}$ is the number of patients in each analysis after omission of patients with missing data,

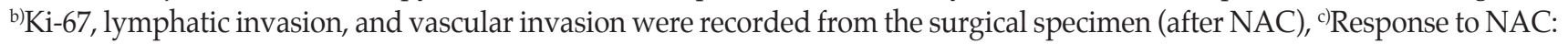
decrease in pathological stage of surgical specimen (after NAC) compared to clinical stage.

phamide, four cycles of doxorubicin and cyclophosphamide followed by four cycles of either docetaxel or paclitaxel, six cycles of doxorubicin and docetaxel, four cycles of paclitaxel and gemcitabine, and six cycles of paclitaxel, gemcitabine, and trastuzumab or lapatinib. Anti-HER2 therapy containing NAC was not yet approved for use by the national health insurance scheme during our study period, and only 29 patients received anti-HER2 containing NAC therapy, mostly under the protocols of clinical trials $[17,18]$. AntiHER2 therapy consisted of trastuzumab or lapatinib [19] for a total duration of 1 year, irrespective of whether it was administered as neoadjuvant or adjuvant therapy. All patients who underwent conservative breast surgery and patients with clinical tumor sizes $\geq 5 \mathrm{~cm}$ and/or clinical N2 and N3 disease were offered adjuvant radiation therapy. Adjuvant endocrine therapy was also offered to all patients with HR positive tumors at the discretion of the physician in charge.

\section{Statistical analysis}

All statistical analyses were performed using STATA ver. 10 (StataCorp LP, College Station, TX). Differences in categorical variables were analyzed using chi-square test, and differences in continuous variables were evaluated using analysis of variance. A pathological complete response (pCR) was defined as the absence of any invasive or in-situ tumor on pathological examination, both in the primary breast and in the lymph nodes. This definition was in accordance with the international expert panel recommendations published by Kaufmann et al. [20]. RFS was defined as the time from the date of diagnosis to the date of local or distant recurrence. OS was defined as the time from the date of diagnosis to death from any cause, or to the date of the last follow-up for living patients. RFS and OS curves were plotted using the Kaplan-Meier method, and compared using the log-rank test. Multivariate Cox regression analysis was used to estimate the hazard ratio, the 95\% confidence interval (CI), and the 
Table 2. Change in the molecular subtypes, hormone receptor and HER2 status after NAC

\begin{tabular}{|c|c|c|c|c|c|c|c|c|c|}
\hline \multirow{2}{*}{$\begin{array}{l}\text { Before NAC } \\
(n=290)\end{array}$} & \multicolumn{8}{|c|}{ After NAC } & \multirow{2}{*}{ p-value } \\
\hline & HR+/HER2- & HR+/HER2+ & HR-/HER2+ & TN & HR+ & HR- & HER2+ & HER2- & \\
\hline HR+/HER2- & $140(89.7)$ & 0 & 0 & $16(10.3)$ & - & - & - & - & $<0.001$ \\
\hline HR+/HER $2+$ & $12(21.4)$ & $37(66.1)$ & $6(10.7)$ & $1(1.8)$ & - & - & - & - & \\
\hline HR-/HER2+ & $1(3.8)$ & $10(38.5)$ & $12(46.2)$ & $3(11.5)$ & - & - & - & - & \\
\hline $\mathrm{TN}$ & $18(34.6)$ & 0 & 0 & $34(65.4)$ & - & - & - & - & \\
\hline $\mathrm{HR}+$ & - & - & - & - & $189(89.2)$ & $23(10.8)$ & - & - & $<0.001$ \\
\hline HR- & - & - & - & - & $29(37.2)$ & $49(62.8)$ & - & - & \\
\hline HER2+ & - & - & - & - & - & - & $65(79.3)$ & $17(20.7)$ & $<0.001$ \\
\hline HER2- & - & - & - & - & - & - & 0 & $208(100)$ & \\
\hline
\end{tabular}

Values are presented as number (\%). HER2, human epidermal growth factor 2; NAC, neoadjuvant chemotherapy; HR, hormone receptor; $\mathrm{TN}$, triple negative.

Table 3. Change in estrogen receptor and progesterone receptor Allred scores after neoadjuvant chemotherapy

\begin{tabular}{lccc} 
Change $(\mathrm{n}=\mathbf{2 9 0})$ & Decrease in score & No change & Increase in score \\
ER Allred score change & $69(23.8)$ & $172(59.3)$ & $49(16.9)$ \\
PR Allred score change & $113(39.0)$ & $110(37.9)$ & $67(23.1)$ \\
\hline
\end{tabular}

Values are presented as number (\%). ER, estrogen receptor; PR, progesterone receptor.

effects of the clinical and pathological variables. A two-sided p-value of $<0.05$ was considered statistically significant.

\section{Ethics}

This study was reviewed and approved by the Institutional Review Board of National Cancer Center, Korea.

\section{Results}

\section{Patient characteristics}

Of 4,460 patients who underwent surgery for invasive breast cancer at our institution during the study period, 449 consecutive patients received NAC. Of these patients, 322 had available data on their HR and HER2 status, both from diagnostic core biopsy specimen analysis prior to NAC and the surgical specimen after NAC. The median patient age was 46.0 years (range, 26.0 to 76.0 years), and the median follow-up period was 63.0 months (range, 5.0 to 110.0 months). Clinically, $24.4 \%$ of the tumors were stage II and $75.6 \%$ were stage III. In IHC analysis before NAC, $61.2 \%$ of the tumors were ER positive (ER+) and $61.8 \%$ of the tumors were PR positive (PR+). In total, 242 tumors $(71.2 \%)$ were HR+ at diagnosis. There were 100 HER2+ tumors (31.1\%), and $55 \mathrm{TN}$ tumors $(17.1 \%)$.

A pCR was achieved in 32 patients $(9.9 \%) ; 6.6 \%$ in the HR+ / HER2- group, $13.8 \%$ in the HR+/HER $2+$ group, $25.7 \%$ in the HR-/HER2+ group, and $5.5 \%$ in the TN group. These patients were excluded in further analysis.

\section{Changes in molecular subtypes after NAC}

Of the 332 tumors, $167(51.9 \%)$ were HR+/HER2-, 65 $(20.2 \%)$ were HR+/HER2+, $35(10.9 \%)$ were HR-/HER2+, and $55(17.1 \%)$ were $\mathrm{TN}$. The patient and tumor characteristics according to the 4 molecular subtypes are shown in Table 1. Subtype changes occurred in $23.1 \%$ of tumors treated with NAC (Table 2). HR+/HER2- tumors tended to lose their HR expression (10.3\%) to become TN, and TN tumors, in contrast, frequently gained HR expression to become HR+/HER2- (34.6\%). In addition, HR+/HER2+ tumors showed a higher tendency to lose their HER2 positivity $(21.4 \%)$ than their HR positivity (10.7\%). HR-/HER2+ tumors gained HR positivity to form HR+/HER2+ tumors $(38.5 \%)$ more often than they lost HER2 overexpression to become TN tumors. 

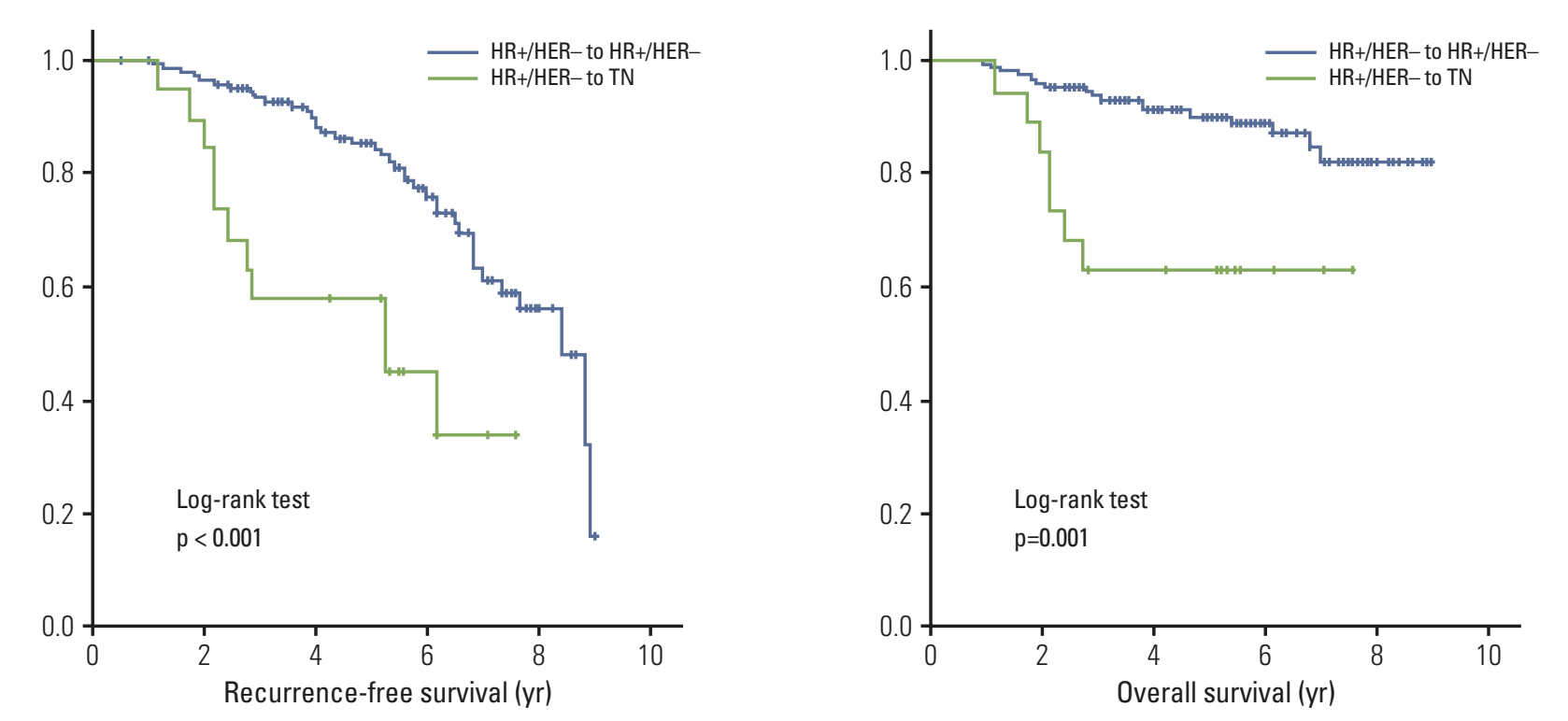

A
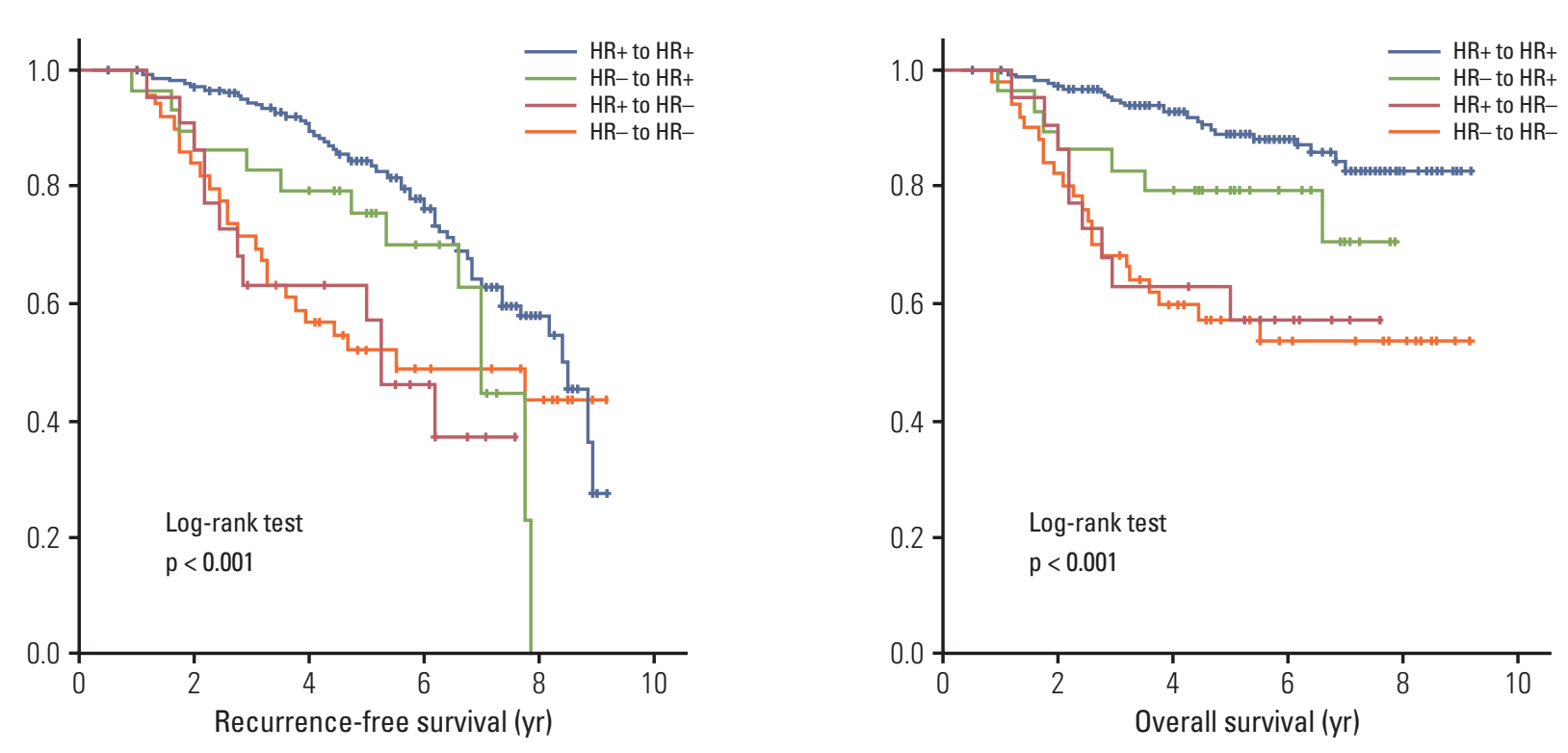

Fig. 1. Kaplan-Meier plots of recurrence free survival and overall survival for HR+/HER- tumors which remained unchanged vs. those which turned to TN (A) and HR status changes (B). HR, hormone receptor; HER2, human epidermal growth factor 2; TN, triple negative.

\section{Changes in the HR status, HER2 status, ER and PR Allred scores after NAC}

In total, 52 tumors $(17.9 \%)$ underwent HR status conversions, and 17 tumors (5.9\%) showed changes in HER2 status. Regarding the HR status, the change from HR- to HR+ was significantly more frequent than the change from $\mathrm{HR}+$ to
HR- $(37.2 \%$ vs. $10.8 \%$; $<$ < 0.001). While no tumors of the HER2- phenotype changed to HER2+ after NAC, 20.7\% of HER2+ tumors became HER2- tumors (Table 2). ER Allred scores showed more stability than PR Allred scores, with PR Allred scores showing a tendency to decrease after NAC (Table 3). 
Table 4. Analysis of recurrence-free survival with clinical characteristics and molecular subtype changes

\begin{tabular}{|c|c|c|c|c|}
\hline \multirow{2}{*}{ Factor } & \multicolumn{2}{|c|}{ Univariate } & \multicolumn{2}{|c|}{ Multivariate $^{\mathrm{a})}$} \\
\hline & Hazard ratio $(95 \% \mathrm{CI})$ & p-value & Hazard ratio $(95 \% \mathrm{CI})$ & p-value \\
\hline \multicolumn{5}{|l|}{ Age (yr) } \\
\hline$<45$ & 1 & 0.678 & - & - \\
\hline$\geq 45$ & $0.92(0.64-1.34)$ & & - & \\
\hline \multicolumn{5}{|l|}{ Clinical stage } \\
\hline Stage II & 1 & 0.003 & 1 & 0.001 \\
\hline Stage III & $2.23(1.32-3.76)$ & & $2.93(1.54-5.58)$ & \\
\hline \multicolumn{5}{|l|}{ Grade } \\
\hline Grade 1 or 2 & 1 & 0.035 & 1 & 0.889 \\
\hline Grade 3 & $1.52(1.03-2.25)$ & & $1.04(0.63-1.72)$ & \\
\hline \multicolumn{5}{|l|}{$\mathrm{Ki}-67(\%)^{\mathrm{b})}$} \\
\hline$<15$ & 1 & 0.002 & 1 & 0.030 \\
\hline$\geq 15$ & $1.85(1.26-2.73)$ & & $1.70(1.05-2.74)$ & \\
\hline \multicolumn{5}{|l|}{ Response to NAC ${ }^{c}$} \\
\hline Yes & 1 & 0.011 & 1 & 0.016 \\
\hline No & $1.65(1.12-2.42)$ & & $1.71(1.11-2.63)$ & \\
\hline \multicolumn{5}{|l|}{ Molecular subtype conversions } \\
\hline HR+ / HER2- to HR+/HER2- & 1 & & 1 & \\
\hline $\mathrm{HR}+/ \mathrm{HER} 2-$ to TN & $3.62(1.84-7.10)$ & 0.001 & $3.54(1.60-7.85)$ & 0.002 \\
\hline $\mathrm{HR}+/ \mathrm{HER} 2+$ to $\mathrm{HR}+/ \mathrm{HER} 2+$ & $0.97(0.52-1.82)$ & 0.924 & $0.94(0.49-1.82)$ & 0.862 \\
\hline HR+ / HER2 + to HR + / HER2- & $0.73(0.23-2.38)$ & 0.604 & $0.62(0.15-2.65)$ & 0.521 \\
\hline HR-/HER2 + to HR-/HER2+ & $1.16(0.45-2.96)$ & 0.758 & $1.06(0.35-3.22)$ & 0.921 \\
\hline HR-/HER2 + to HR+/HER2+ & $1.24(0.44-3.48)$ & 0.683 & $1.06(0.36-3.11)$ & 0.921 \\
\hline $\mathrm{TN}$ to $\mathrm{TN}$ & $3.28(1.89-5.70)$ & $<0.001$ & $3.70(1.86-7.36)$ & 0.001 \\
\hline TN to HR+/HER2- & $2.25(1.09-4.67)$ & 0.029 & $1.59(0.63-4.02)$ & 0.324 \\
\hline
\end{tabular}

$\mathrm{CI}$, confidence interval; NAC, neoadjuvant chemotherapy; HR, hormone receptor; HER2, human epidermal growth factor 2; $\mathrm{TN}$, triple negative. ${ }^{a}$ Multivariate analysis was performed with covariates of clinical stage, grade, Ki-67 and response to NAC, $\left.{ }^{b}\right) \mathrm{Ki}-67$ was recorded from the surgical specimen (after NAC), c'Response to NAC: decrease in pathological stage of surgical specimen (after NAC) compared to clinical stage.

\section{Molecular subtype conversions and clinical outcomes}

The 5-year RFS rates of the HR+/HER2-, HR+/HER2+, HR-/HER2+, and TN groups at diagnosis were $76 \%, 84 \%$, $85 \%$, and $48 \%$ respectively, and the 5-year OS rates for these groups were $86 \%, 85 \%, 85 \%$, and $59 \%$ respectively. These differences were statistically significant $(\mathrm{p}<0.001)$.

The RFS and OS of patients with the more common molecular subtype conversions were analyzed. Compared to patients who maintained a molecular subtype of $\mathrm{HR}+$ / HER2- tumors before and after NAC, those who underwent conversion from HR+ / HER2- to the TN type showed significantly worse prognosis (Fig. 1). In multivariate analysis with covariates of tumor grade, clinical stage, Ki-67 after NAC and response to NAC, this change continued to demonstrate a significantly worse outcome in terms of both RFS (hazard ratio, 3.54; $95 \%$ CI, 1.60 to 7.85 ) and OS (hazard ratio, 3.73;
95\% CI, 1.34 to 10.38). In contrast to the $\mathrm{TN}$ to $\mathrm{TN}$ group, patients who converted from TN to HR+/HER2- showed better RFS and OS, which was not significantly different from that of the HR+/HER2- group (Tables 4 and 5).

\section{HR status conversions, ER and PR Allred score changes and clinical outcomes}

Changes in HR status after NAC were classified as: $\mathrm{HR}+$ to $\mathrm{HR}+, \mathrm{HR}+$ to HR-, HR- to HR-, and HR- to HR+. RFS and OS differed significantly among the four groups $(\mathrm{p}<0.001)$ (Fig. 1). In multivariate analysis with covariates of tumor grade, clinical stage, Ki-67 after NAC and response to NAC, designating the $\mathrm{HR}+$ to $\mathrm{HR}+$ group as reference, patients who converted from $\mathrm{HR}+$ to HR- status had significantly worse RFS (hazard ratio, 2.38; 95\% CI, 1.17 to 4.84 ) and OS (hazard ratio, 2.66; 95\% CI, 1.11 to 6.39). Patients who had 
Table 5. Analysis of overall survival with clinical characteristics and molecular subtype changes

\begin{tabular}{|c|c|c|c|c|}
\hline \multirow{2}{*}{ Factor } & \multicolumn{2}{|c|}{ Univariate } & \multicolumn{2}{|c|}{ Multivariate $^{\mathrm{a})}$} \\
\hline & Hazard ratio $(95 \% \mathrm{CI})$ & p-value & Hazard ratio $(95 \% \mathrm{CI})$ & p-value \\
\hline \multicolumn{5}{|l|}{ Age (yr) } \\
\hline$<45$ & 1 & 0.543 & - & - \\
\hline$\geq 45$ & $0.86(0.54-1.38)$ & & - & \\
\hline \multicolumn{5}{|l|}{ Clinical stage } \\
\hline Stage II & 1 & 0.001 & 1 & 0.004 \\
\hline Stage III & $5.45(1.98-15.02)$ & & $5.72(1.74-18.81)$ & \\
\hline \multicolumn{5}{|l|}{ Grade } \\
\hline Grade 1 or 2 & 1 & 0.012 & 1 & 0.702 \\
\hline Grade 3 & $2.04(1.17-3.55)$ & & $1.13(0.60-2.13)$ & \\
\hline \multicolumn{5}{|l|}{$\mathrm{Ki}-67(\%)^{\mathrm{b})}$} \\
\hline$<15$ & 1 & $<0.001$ & 1 & 0.066 \\
\hline$\geq 15$ & $3.01(1.78-5.11)$ & & $1.86(0.96-3.60)$ & \\
\hline \multicolumn{5}{|l|}{ Response to $\mathrm{NAC}^{\mathrm{c})}$} \\
\hline Yes & 1 & 0.012 & 1 & 0.013 \\
\hline No & $1.91(1.15-3.15)$ & & $2.06(1.17-3.65)$ & \\
\hline \multicolumn{5}{|l|}{ Molecular subtype conversions } \\
\hline HR+ / HER2- to HR+ / HER2- & 1 & & 1 & \\
\hline $\mathrm{HR}+/ \mathrm{HER} 2-$ to TN & $4.02(1.65-9.79)$ & 0.002 & $3.73(1.34-10.38)$ & 0.012 \\
\hline HR+ / HER2+ to HR+ / HER2+ & $1.07(0.39-2.92)$ & 0.897 & $1.20(0.42-3.38)$ & 0.737 \\
\hline HR+ / HER2 + to HR+ / HER2- & $1.28(0.29-5.55)$ & 0.746 & $1.02(0.13-8.00)$ & 0.984 \\
\hline HR-/HER2 + to HR-/HER2+ & $2.75(0.92-8.22)$ & 0.071 & $2.50(0.77-8.18)$ & 0.129 \\
\hline HR-/HER2+ to HR+/HER2+ & $0.77(0.10-5.79)$ & 0.797 & $0.70(0.09-5.52)$ & 0.736 \\
\hline $\mathrm{TN}$ to $\mathrm{TN}$ & $6.30(3.17-12.50)$ & $<0.001$ & $5.85(2.53-13.51)$ & $<0.001$ \\
\hline TN to HR+/HER2- & $3.22(1.26-8.22)$ & 0.015 & $2.74(0.92-8.19)$ & 0.071 \\
\hline
\end{tabular}

$\mathrm{CI}$, confidence interval; NAC, neoadjuvant chemotherapy; HR, hormone receptor; HER2, human epidermal growth factor 2; $\mathrm{TN}$, triple negative. ${ }^{\mathrm{a})}$ Multivariate analysis was performed with covariates of clinical stage, grade, Ki-67 and response to NAC, ${ }^{b} \mathrm{Ki}-67$ was recorded from the surgical specimen (after NAC), cResponse to NAC: decrease in pathological stage of surgical specimen (after NAC) compared to clinical stage.

HR- to HR+ conversions appeared to have better RFS (hazard ratio, 1.32; 95\% CI, 0.64 to 2.74 ) and OS (hazard ratio, $1.64 ; 95 \% \mathrm{CI}, 0.23$ to 4.30 ) than those who remained HR-(for RFS: hazard ratio, 2.01; 95\% CI, 1.10 to 3.69; for OS: hazard ratio, $3.68 ; 95 \% \mathrm{CI}, 1.80$ to 7.49 ). RFS and OS did not differ significantly with changes in the HER2 status or ER and PR Allred score after NAC (data not shown).

\section{Discussion}

We found that breast tumors underwent frequent molecular subtype conversions after NAC. The rate of change in HR status noted in our study $(17.9 \%)$ is in agreement with the rates reported by Tacca et al. [6] and Hirata et al. [7] (16\% and $23 \%$, respectively). In addition, HER2 status tended to change less frequently than the HR status in response to NAC, as reported in other studies [5,7].

According to our results, changes in the molecular subtypes of tumors after NAC could alter the patient prognosis. In our study, HR+/HER2- patients who converted to TN had a significantly shorter RFS and OS than those who remained as HR+/HER2-, and both RFS and OS of those who converted from TN to HR+/HER2- improved compared to those who remained as TN. Although all patients with HR+ status were treated with endocrine therapy, the outcomes of patients who converted from $\mathrm{HR}+$ to HR- status were also worse than those who remained HR positive. In agreement with our findings, Tacca et al. [6] found that patients with a change from HR- tumors to HR+ tumors after NAC had better disease-free survival (DFS) and OS than those in whom the status of HR- tumors did not change. In 
addition, Chen et al. [21] reported that patients who switched from HR+ to HR- status had poorer DFS and OS when compared to those who remained HR+; they also found that adjuvant endocrine therapy did not confer a survival benefit in patients in whom the status of HR+ tumors changed to HR-. In contrast, Hirata et al. [7], showed that endocrine therapy significantly improves the DFS and OS in patients who had HR status conversions.

To the best of our knowledge, this is the first study exploring the changes in the HR/HER2 subtypes before and after NAC. This subtype grouping provides more specific information regarding the biologic and behavioral characteristics of tumors than the individual consideration of the ER, PR, or HER2 status. Although the patient numbers in most of the molecular subtype conversion groups were small, this study provides meaningful information on the frequency and trends in the changes of each subtype, as well as the clinical impact of the molecular subtype conversions.

We acknowledge that this study had some limitations. First, it was retrospective in nature, although our breast cancer database is prospectively maintained. Therefore, it was not possible to control for the type of NAC or adjuvant treatment that the patients received, or to exclude selection bias. There were also some missing data in our study, which is an inherent problem in retrospective studies. However, as the amount of missing data is small we do not expect the results to be significantly affected. Second, we classified the molecular subtype of tumors only according to the HR and HER2 status, and our molecular subtype approximation would have correlated better with the four major molecular subtypes (luminal A and B, HER and basal-like), if we had other available data on the core biopsy specimens such as Ki-67, cytokeratin $5 / 6$ or HER1 [12,13,22]. However, our method is a simple and convenient way to estimate a tumor's subtype and involves the use of biomarkers that are routinely tested in clinical practice. In addition, its usefulness was previously demonstrated in other studies $[14,23,24]$. Finally, unlike IHC, FISH was performed only once during the course of treat- ment, which could have affected HER2 status determination. We found no statistically significant difference in the long term outcomes of patients who showed changes in their HER2 status, likely because most patients did not receive HER2-targeted treatment during the study period. In the era of HER2-targeted therapy, long term outcomes of patients with HER2 status conversions may differ significantly, and we await further results from future studies on this patient group.

\section{Conclusion}

Molecular subtypes of breast cancers changed frequently after NAC and these changes showed correlation with longterm prognosis. Thus, molecular subtypes should be reassessed routinely after NAC using surgical specimens. Understanding the patterns of change in each molecular subtype could provide important predictive and prognostic information, as breast cancer management becomes more individualized.

\section{Conflicts of Interest}

Conflict of interest relevant to this article was not reported.

\section{Acknowledgments}

This work was supported by a research grant from the National Cancer Center (NCC-1410690-2), Republic of Korea.

\section{References}

1. Wolmark N, Wang J, Mamounas E, Bryant J, Fisher B. Preoperative chemotherapy in patients with operable breast cancer: nine-year results from National Surgical Adjuvant Breast and Bowel Project B-18. J Natl Cancer Inst Monogr. 2001;(30): 96-102.

2. Chen AM, Meric-Bernstam F, Hunt KK, Thames HD, Oswald MJ, Outlaw ED, et al. Breast conservation after neoadjuvant chemotherapy: the MD Anderson Cancer Center experience. J Clin Oncol. 2004;22:2303-12.
3. Mittendorf EA, Buchholz TA, Tucker SL, Meric-Bernstam F, Kuerer HM, Gonzalez-Angulo AM, et al. Impact of chemotherapy sequencing on local-regional failure risk in breast cancer patients undergoing breast-conserving therapy. Ann Surg. 2013;257:173-9.

4. Zhang N, Moran MS, Huo Q, Haffty BG, Yang Q. The hormonal receptor status in breast cancer can be altered by neoadjuvant chemotherapy: a meta-analysis. Cancer Invest. 2011;29: 594-8. 
5. van de Ven S, Smit VT, Dekker TJ, Nortier JW, Kroep JR. Discordances in ER, PR and HER2 receptors after neoadjuvant chemotherapy in breast cancer. Cancer Treat Rev. 2011;37: 422-30.

6. Tacca O, Penault-Llorca F, Abrial C, Mouret-Reynier MA, Raoelfils I, Durando X, et al. Changes in and prognostic value of hormone receptor status in a series of operable breast cancer patients treated with neoadjuvant chemotherapy. Oncologist. 2007;12:636-43.

7. Hirata T, Shimizu C, Yonemori K, Hirakawa A, Kouno T, Tamura K, et al. Change in the hormone receptor status following administration of neoadjuvant chemotherapy and its impact on the long-term outcome in patients with primary breast cancer. Br J Cancer. 2009;101:1529-36.

8. Davies C, Pan H, Godwin J, Gray R, Arriagada R, Raina V, et al. Long-term effects of continuing adjuvant tamoxifen to 10 years versus stopping at 5 years after diagnosis of oestrogen receptor-positive breast cancer: ATLAS, a randomised trial. Lancet. 2013;381:805-16.

9. Zardavas D, Ades F, de Azambuja E. Clinical practice-changing trials: the HERA study paradigm. Expert Rev Anticancer Ther. 2013;13:1249-56.

10. Rouzier R, Perou CM, Symmans WF, Ibrahim N, Cristofanilli $\mathrm{M}$, Anderson $\mathrm{K}$, et al. Breast cancer molecular subtypes respond differently to preoperative chemotherapy. Clin Cancer Res. 2005;11:5678-85.

11. von Minckwitz G, Fontanella C. Selecting the neoadjuvant treatment by molecular subtype: how to maximize the benefit? Breast. 2013;22 Suppl 2:S149-51.

12. Schnitt SJ. Classification and prognosis of invasive breast cancer: from morphology to molecular taxonomy. Mod Pathol. 2010;23 Suppl 2:S60-4.

13. Carey LA, Perou CM, Livasy CA, Dressler LG, Cowan D, Conway $\mathrm{K}$, et al. Race, breast cancer subtypes, and survival in the Carolina Breast Cancer Study. JAMA. 2006;295:2492-502.

14. Onitilo AA, Engel JM, Greenlee RT, Mukesh BN. Breast cancer subtypes based on ER/PR and Her2 expression: comparison of clinicopathologic features and survival. Clin Med Res. 2009;7:4-13.

15. Lee S, Kim SW, Kim SK, Lee KS, Kim EA, Kwon Y, et al.
Locoregional recurrence of breast conserving surgery after preoperative chemotherapy in korean women with locally advanced breast cancer. J Breast Cancer. 2011;14:289-95.

16. Lee KS, Ro J, Lee ES, Kang HS, Kim SW, Nam BH, et al. Primary systemic therapy with intermittent weekly paclitaxel plus gemcitabine in patients with stage II and III breast cancer: a phase II trial. Invest New Drugs. 2010;28:83-90.

17. Im SA, Lee KS, Ro J, Lee ES, Kwon Y, Ahn JH, et al. Phase II trial of preoperative paclitaxel, gemcitabine, and trastuzumab combination therapy in HER2 positive stage II / III breast cancer: the Korean Cancer Study Group BR 07-01. Breast Cancer Res Treat. 2012;132:589-600.

18. Park IH, Lee KS, Kang HS, Kim SW, Lee S, Jung SY, et al. A phase Ib study of preoperative lapatinib, paclitaxel, and gemcitabine combination therapy in women with HER2 positive early breast cancer. Invest New Drugs. 2012;30:1972-7.

19. Tomasello G, de Azambuja E, Dinh P, Snoj N, Piccart-Gebhart M. Jumping higher: is it still possible? The ALTTO trial challenge. Expert Rev Anticancer Ther. 2008;8:1883-90.

20. Kaufmann M, Hortobagyi GN, Goldhirsch A, Scholl S, Makris A, Valagussa P, et al. Recommendations from an international expert panel on the use of neoadjuvant (primary) systemic treatment of operable breast cancer: an update. J Clin Oncol. 2006;24:1940-9.

21. Chen S, Chen CM, Yu KD, Zhou RJ, Shao ZM. Prognostic value of a positive-to-negative change in hormone receptor status after neoadjuvant chemotherapy in patients with hormone receptor-positive breast cancer. Ann Surg Oncol. 2012; 19:3002-11.

22. Voduc KD, Cheang MC, Tyldesley S, Gelmon K, Nielsen TO, Kennecke H. Breast cancer subtypes and the risk of local and regional relapse. J Clin Oncol. 2010;28:1684-91.

23. Houssami N, Macaskill P, von Minckwitz G, Marinovich ML, Mamounas E. Meta-analysis of the association of breast cancer subtype and pathologic complete response to neoadjuvant chemotherapy. Eur J Cancer. 2012;48:3342-54.

24. Kawajiri H, Takashima T, Aomatsu N, Kashiwagi S, Noda S, Onoda N, et al. Prognostic significance of pathological complete response following neoadjuvant chemotherapy for operable breast cancer. Oncol Lett. 2014;7:663-8. 